\title{
Molecular principles of cancer invasion and metastasis (Review)
}

\author{
MATHIAS FELIX LEBER ${ }^{1}$ and THOMAS EFFERTH ${ }^{2}$ \\ ${ }^{1}$ University of Mainz, Mainz; ${ }^{2}$ German Cancer Research Center, \\ Pharmaceutical Biology (C015), Heidelberg, Germany
}

Received October 2, 2008; Accepted November 29, 2008

DOI: 10.3892/ijo_00000214

\begin{abstract}
The main threat and the reason for most cancer deaths are not the primary neoplasias, but secondary tumors, the metastases. Drastic phenotypic and biochemical changes occur during the metamorphosis of a normal tissue cell into an invasive cancer cell. These alterations concern various areas such as growth factor signaling, cell-cell adhesion, gene expression, motility or cell shape. Cancer cells of epithelial origin can even shed their typical qualities and characteristics and adopt a mesenchymal-like phenotype. This is often referred to as an epithelial-mesenchymal transition. Various oncogenes, tumor suppressor genes and metastasis suppressor genes are known to affect the invasiveness and the metastatic potential of tumor cells. Cells of the innate and adaptive immunity, adjacent stroma cells as well as chemokines and their receptors also play a vital role in the spread of cancer cells. Furthermore, the micro-environment, vascularization and the supply with special cytokines affect the above-mentioned changes. Finally, some researchers claim that tumors consist of two types of cells - transit amplifying cells and cancer stem cells. Only the latter are thought to be able to proliferate indefinitely and thus they might be the cells that successfully spread and initially build most of the clinically relevant metastases. This review article describes some of the molecular principles which underlie those changes as well as covers some aspects of current research.
\end{abstract}

\section{Contents}

1. Introduction

2. Molecular principles of cancer invasion and metastasis

3. Conclusion

Correspondence to: Dr Thomas Efferth, German Cancer Research Center, Pharmaceutical Biology (C015), Im Neuenheimer Feld 280, D-69120 Heidelberg, Germany

E-mail: t.efferth@dkfz.de

Key words: apoptosis, cancer, invasion, metastasis, neoangiogenesis, signal transduction

\section{Introduction}

In the course of carcinogenesis and the associated dedifferentiation of a healthy cell to a malignant, anaplastic tumor, the cell must acquire the following capabilities (1).

Cell division in the absence of external growth stimulatory signals. Typically, cells in healthy tissues only divide if they receive growth stimulatory signals from other cells in the form of growth factors. These are detected in the extracellular matrix (ECM) by growth factor receptors such as vascular endothelial growth factor receptor (VEGFR) or epidermal growth factor receptor (EGFR). Both are transmembrane proteins and belong to the family of receptor tyrosine kinases (RTKs). When EGFR binds to one of its ligands (EGF, Her2, Neu, ErbB2), it activates certain signal transduction pathways such as the Ras/MAPK (rat sarcoma/mitogen activated protein kinase), Akt/PKB (protein kinase B) or STAT-3 (signal transducer and activator of transcription) pathways which in turn favor proliferation or inhibit apoptosis (2).

In contrast, tumor cells are no longer dependent on exogenous growth factors. For example, they evade this dependence through the endogenous production of their own mitogenic factors which they secrete into the ECM. This autocrine signaling finally stimulates proliferation.

Further strategies are the over-expression of receptor tyrosine kinases on the surface of the tumor cell or the formation of structurally altered RTKs which activate the signal transduction pathways even in the absence of mitogenic factors.

Another possibility directly involves the cytoplasmatic components of the signaling pathway. The Ras oncoprotein, which participates in the signaling pathways mentioned above, normally resides in a quiescent state in the absence of growth factors. In cancer cells though, the Ras oncogene is mutated in about $20 \%$ (3) of all cases and permanently conveys growth stimulatory signals. All these strategies and mechanisms enable neoplastic cells to proliferate even in the absence of exogenous growth factors.

Growth in spite of exogenous growth inhibitory signals. Whenever cells divide to build up a tissue or an organ, they will come across other cells or tissues, due to the narrowness of the body. In order to protect the structural integrity of these tissues or organs and thus their function in the body, those cells will convey growth inhibitory signals. This mechanism, which is called contact-dependent inhibition of 
growth (4) or simply contact inhibition, is crucial for the viability of all higher organisms. Tumor cells, however, have lost their contact inhibition and are able to divide even in the presence of growth inhibitory signals (1). Consequently, neoplasias can thrust aside, invade, and ultimately destroy other tissues through their intense proliferation.

Evasion of apoptosis. Within the cell, there are several mechanisms and proteins which detect damage to the DNA or the metabolism and eventually take certain counteractions. Probably the most famous example is the tumor suppressor gene TP53, which encodes for the protein p53 also referred to as the 'guardian of the genome' (5). TP53 is the gene most often mutated in tumors $(\sim 50 \%)(6)$. Therefore, p53 is an attractive target for the development of novel therapeutics such as Leptomycin-B or Nutlin-3, which inhibit the interaction of p53 and mdm-2 (p53-inhibitor) (7).

A heterozygous germ line mutation of the TP53 gene (located at chromosome 17p13) causes the Li-Fraumeni syndrome, which is accompanied with multiple tumors in early life (8). As a result of certain factors such as hypoxia, UV radiation, DNA damage, or transcription blockades, the p53 concentration increases rapidly and activates the p53 signaling pathway.

This is accomplished through the phosphorylation of p53 by Chk 2 which inhibits the interaction of p53 and $\mathrm{mdm} 2$ and the following ubiquitination of p53. Thus, it is not degraded by the proteasome, and its concentration increases. P53 operates as a transcription factor of several genes such as p21/CDKN1A which inhibits cyclin/cdk complexes. These are essential for the progress of the cell cycle and thus for proliferation. In that way, p21 leads to cell cycle arrest.

Depending on the severity of damage, the cell cycle is either blocked through the increase of p53 concentration and distinct repair mechanisms are activated (such as nucleotideexcision, mismatch repair, direct reversal, recombination repair, base-excision), or apoptosis is induced. Apoptosis (programmed cell death, PCD) is a well-regulated and controlled process, which can be triggered via extrinsic/ receptor-based pathways, through binding of the FAS ligand to its receptor (CD95, FAS, APO-1) and activation of the death inducing signal complex (DISC) or through TNF- $\alpha$ / TRAIL-receptors and their ligands. Apoptosis can also be induced by the intrinsic/mitochondrial pathway which is not receptor based, and results in the release of cytochrome $c$ out of the mitochondria and the formation of the apoptosome. In the terminal step, executioner/effector-caspases (cysteinyl aspartate specific proteases) degrade miscellaneous cellular substrates such as lamin (intermediate filament in the nuclear lamina) or actin (cytoskeleton) and thereby lead to cell death. Additionally, caspase activated deoxyribonuclease (CAD) is activated, which cleaves the DNA endonucleolytically (9).

The two pathways should not be considered as completely distinct, since activation of the extrinsic pathway is capable of stimulating the intrinsic pathway via special mediators (e.g., the protein Bid 'BH3 interacting domain death agonist') (10). Characteristic for apoptosis is the occurrence of small membrane blebs filled with degraded fragments of the cytoplasm (membrane blebbing), which are incorporated by the surrounding cells, and the absence of an inflammatory environment (in contrast to necrosis). Cancer cells have developed several strategies to evade apoptosis even under conditions, which normally activate it. Those changes contain mutations in the $C D 95 / F A S$ gene, gene silencing by methylation of its promoter, increased synthesis of antiapoptotic factors (Bcl-2, FLIPs, IAPs, e.g., survivin), loss-offunction mutations in pro-apoptotic genes (such as Bax), and a controversial hypothesis called 'tumor counter attack'. Here, CD8 ${ }^{+}-\mathrm{T}$-lymphocytes, which should assault the tumor, are driven into apoptosis by the cancer cell through the increased expression of the CD95 ligand either on the surface of the cell or soluble (11).

Neoangiogenesis. Tissues which are particularly active in their metabolism such as rapidly proliferating cells need an adequate supply of nutrients and oxygen, as well as a continuous removal of their partly cytotoxic metabolic waste products. Tumors are often intensely proliferating tissues and are, therefore, dependent on a sufficient connection to the vascular system.

The term neoangiogenesis describes the process of tumor vascularization that is the formation of small capillaries. A tumor can only proliferate up to the size of $1-2 \mathrm{~mm}\left(\sim 10^{7}\right.$ cells $)$ without own vascularization. If a tumor does not possess sufficient vascularization, hypoxia occurs and induces the expression of several pro-angiogenic factors. The balance between pro- and anti-angiogenic factors regulates tumor vascularization. Amongst others, VEGF, platelet-derived growth factor (PDGF), fibroblast growth factor (FGF), angiostatin, endostatin and thrombospondins influence angiogenesis (12). These angiogenic factors are secreted by the tumor and lead to a concentration gradient which drives angiogenesis. In addition, ephrin receptor signaling seems to play a vital role in angiogenesis since the blockage of EphA2/EphA3 receptor signaling leads to inhibition of angiogenesis in vivo and in vitro (13).

Potential immortalization. Normal human cells in cell culture die after $\sim 50$ mitoses (Hayflick limit) (14). The main reason for this behavior is the loss of genetic stability due to progressive telomere shortening. Telomeres represent the linear ends of the chromosomes which shorten by $\sim 60 \mathrm{bp}(\leq 200 \mathrm{bp}$ ) per cell division due to a replication problem (15). Without the telomeres, the DNA can not be replicated accurately, chromosome fusions occur and the cell finally enters the stadium of replicative senescence (16), which can be detected by the expression of senescence-associated $\beta$-galactosidase (17). By different mechanisms, telomere shortening, reactive oxygen species (ROS) and DNA mutations all contribute to cell aging. Consequently, the cells of a tumor should go into senescence or undergo apoptosis after a certain amount of cell divisions at least due to telomere shortening. Neoplastic cells, however, develop counteractions. For example, they activate the enzyme telomerase, a reverse transcriptase, which elongates the telomeres. A RNA matrix (hTR, human telomerase RNA), which is part of the telomerase, is used as a template for the TTAGGG-hexanucleotide-repeats (in humans) which compose the telomeres. This is commonly referred to as a ribonucleoprotein (RNP). In general, the telomerase consists of two parts of hTR, hTERT (human 
telomerase reverse transcriptase, catalytically active subunit of the telomerase) and dyskerin (associated protein) each (18). Typically, the telomerase is not active in somatic cells (or more precisely expressed at low levels), but highly active in germ-, stem-, and most cancer cells. Activation of telomerase leads to potential immortalization of those cells (16), whereas inhibition of telomerase in tumor cells normally leads to senescence or apoptosis $(15,19,20)$. For reasons mentioned above, the telomerase is point of attack for several therapeutics which inhibit its catalytic activity (20). For example, special antisense RNA oligonucleotide molecules have been engineered which selectively bind to the RNA-template of the telomerase (21). Furthermore, there are therapeutics which inhibit the reverse transcriptase activity of hTERT as well as molecules which drive the formation of G-quadruplex structures (22). Those are special foldings of the guanine-rich region which can be found in the $3^{\prime}$ overhang of the telomeres that inhibit the catalytic activity of hTERT. Besides this main mechanism, another telomere-lengthening mechanism has been discovered: alternative lengthening of telomeres (ALT) (23). This represents a telomerase-independent inter-chromosomal copy mechanism. The telomere of a chromosome is used as a template for the elongation of the telomere of yet another chromosome. The ALT mechanism can frequently be found in osteosarcomas, soft tissue sarcomas and glioblastomas.

Invasion and metastasis. Another important capability which cancer cells must acquire accounts for the difference between benign and malignant tumors, invasion and metastasis. A tumor which has not yet reached an invasive phenotype is often referred to as 'carcinoma in situ'. Invasiveness does not only mean the ability to thrust aside adjacent tissues, but also to actively invade and, therefore, destroy these tissues. Metastasis refers to the capability to leave a primary tumor, travel via the circulation to a distant tissue site, and form a secondary tumor. Metastasis consists of five steps which are collectively termed the metastatic cascade (Fig. 1).

1) Invasion and migration: individual cells detach themselves from the primary tumor and invade adjacent, healthy tissue. During this process, several lytic enzymes are secreted which degrade the ECM (extracellular matrix) and, therefore, facilitate migration.

2) Intravasation: the intrusion of cancer cells into the blood and lymphatic vessels. After the attachment on the endothelial cells via adhesion molecules, the neoplastic cells secrete proteolytic enzymes which enable them to infiltrate the blood vessel.

3) Circulation: the aberrant cell travels via the blood stream and has to withstand the conditions present in the blood. These are toxic for cancer cells due to the high concentration of oxygen and cytotoxic lymphocytes. A selection for particularly resistant and aggressive tumor cells takes place.

4) Extravasation: the cells often get stuck in the capillaries of an organ and leave the blood stream by penetrating the endothelium through proliferation and/or proteolytic enzymes.

5) Colonization, proliferation and angiogenesis: the neoplastic cell settles at a distant tissue site and builds a secondary tumor. The latter proliferates and induces neoangiogenesis in order to ensure sufficient vascularization.
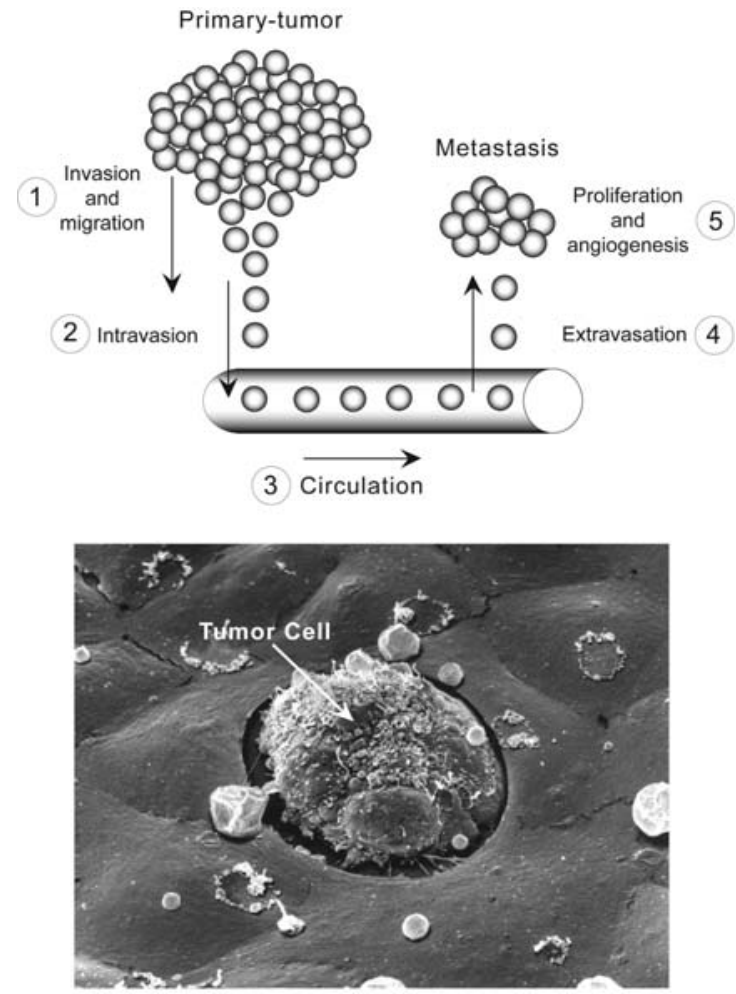

figure 1

Figure 1. Metastasis of tumors. (a) The five steps of the metastatic cascade. (b) Metastatic tumor cell crossing the endothelial cell layer of a blood vessel (electron microscopic image kindly provided by Dr Eberhardt Spiess, German Cancer Research Center, Heidelberg, Germany). Taken from Efferth (46) with permission of Springer, Heidelberg, Germany.

\section{Molecular principles of cancer invasion and metastasis}

It is not the primary tumors, but metastases that are accountable for $\sim 90 \%$ of all cancer deaths (24). Nevertheless, the processes of invasion and metastasis are still insufficiently understood. For example, the question is still unanswered, why some cancers, such as melanomas, tend to metastasize, whereas others, such as squamous carcinomas of the skin, scarcely form secondary tumors. Furthermore, in spite of miscellaneous investigations, the correlation between the size of a tumor and its potential to metastasize is not sufficiently understood. Even smaller tumors in early stages are able to form micrometastases which can be disseminated throughout the body, but rarely yield in solid and clinically-relevant secondary tumors.

In the field of organ transplantation, micro-metastases still pose a threat. They are hardly detectable due to their small size and can, therefore, easily be transplanted along with the organ to a new patient. Because of immune-suppressive medication of the recipient in order to prevent graft rejection, the development of a cancer with donor origin in the recipient is possible

Micro-metastases can also remain in the body of a cancer patient after successful chemo- or radiotherapy. It is possible that these remaining tumor cells start to proliferate uncontrollably even years after the patient is thought to be cured leading to the development of a new cancer. 
Fortunately, the process of metastasis is not very efficient a phenomenon which is often referred to as 'metastatic inefficiency' (25).

Up to one million cells per day can leave a primary mouse tumor of only $1 \mathrm{~g}$ mass $\left(\sim 10^{9}\right.$ cells $)$ and enter the invasion metastasis cascade (26). In direct contrast, the amount of micro-metastases which evolve into clinically relevant secondary tumors is negligible. For possible causes for these paradox facts see section Monoclonality and cancer stem cell theory: do all cancer cells share the same abilities?

During the last couple of years, a wealth of data suggested that cancer cells use the very same mechanisms as healthy cells do - the genetic programs, which are induced by transcription factors, are only activated at the wrong time and in the wrong cells. They normally control crucial steps in early embryogenesis. Leukocytes are also able to travel via the blood stream in all tissues and master the mechanisms of extravasation, migration and intravasation. Furthermore, certain cells of the ectoderm acquire the ability not only to move, but also to change their whole phenotype during gastrulation. They shed their epithelial character, adopt a mesenchymal phenotype and establish the mesoderm. The mesoderm is the medium of the three human germ layers (endoderm, mesoderm, and ectoderm) and builds up bones, muscles, connective tissue, kidneys, blood- and lymphatic vessels, chorda, hematopoietic cells, as well as other essential structures.

This process of conversion from an epithelial to a mesenchymal-like phenotype is called epithelial mesenchymal transition (EMT). The EMT plays a crucial role not only in gastrulation but also during the development of a malignant tumor and the processes of wound healing. The molecular and cell biological principles of invasion and metastasis are highly complex but essential for a profound understanding of carcinogenesis. Various genetic, anatomic and physiologic conditions affect metastasis and are collectively referred to as 'metastatic factors'. These include components of the extracellular matrix, integrins, cadherins, cell-cell adhesion molecules (CAMs, cellular adhesion molecules), factors of cellular movement (motility and chemotaxis), metastasis genes and metastasis suppressors, and several proteinases (plasminogen activators, cathepsins, matrix metalloproteinases as well as their inhibitors). Additionally, the nature and localization of the primary tumor as well as angiogenic factors and the immune system should be considered as metastatic factors. This article will cover several of these factors in the following sections. Below, the distinct steps of the invasion metastasis cascade and their molecular principles will be discussed in detail.

Invasion and migration. About $90 \%$ of all human cancers are derived from epithelial tissues and are, thus, by definition carcinomas. Epithelia are single or multi-layer tissues, which cover virtually all inner and outer surface of the metazoan. They are essential for various functions such as mechanical protection, secretion and resorption, protection from infections, stimulus perception or regulation of transpiration. Therefore, epithelia are specialized tissues which show a striking correlation between function and morphology. An epithelium should provide protection from exogenous toxins and infectious organisms. Thus, the cells are tightly connected via 'tight junctions' and possess only little intercellular space. Epithelial tissues are bordered to the ECM by the basement membrane.

A characteristic feature of epithelial cells is their close anchorage in the tissue and, thus, the absence of motility. Besides the above-mentioned tight junctions, the 'adherens junctions' are of particular importance in this context. Those are homotypic cell-cell adhesions i.e., adhesions between two cells of the same type, which are facilitated by calciumdependent transmembrane glycoproteins (27) such as the cadherins. The functional dysregulation of the cadherin family and especially the loss of E-cadherin are commonly observed in invasive cancer cells (28). The E-cadherin gene $\mathrm{CDH}-1$ is also referred to as a metastasis suppressor gene, because its loss induces an invasive phenotype in immortalized cells. Due to the resulting loosening of tissue structure as well as further cell biological changes (amongst others in the cytoskeleton) it is possible that epithelial cells adopt a rather mesenchymal character. The following section will cover the loss of the E-cadherin association as well as the epithelial mesenchymal transition.

Epithelial mesenchymal transition and modified gene expression of cadherins and cytokeratins. The cadherins of two adjacent cells form homodimers which provide a solid association through adherens junctions. Via the intracellular linker proteins $\alpha-, \beta$-catenin and p120 the highly conserved cytoplasmatic tails of the cadherins interact with the cytoskeleton (29).

In this manner, the cadherins together with the catenins represent a connection between the extracellular space and the cytoskeleton (and thus the intracellular machinery which is accountable for changes in cell morphology and motility). Besides the E-cadherin, there is yet another molecule, $\mathrm{N}$-cadherin, which is expressed by mesenchymal cells (in particular fibroblasts). N-cadherin is often up-regulated in cancer cells, whereas E-cadherin is frequently found to be down-regulated. Phenomena like this do not only occur in the context of a tumor EMT, but can also be observed during gastrulation. The modified cadherin expression can be visualized via immunohistochemistry in advanced carcinomas. Fluorescently-labeled antibodies can be used to detect E-cadherin molecules. By expressing $\mathrm{N}$-cadherin molecules on the surface of carcinoma cells, their affinity to stroma cells, which naturally express $\mathrm{N}$-cadherin, is increased. Hence, the integration of $\mathrm{N}$-cadherin into the cytoplasmatic membrane enhances the migration of the cell from the epithelium towards the stroma. It is exactly this mechanism that contributes to the development of malignant melanomas. Their cells are able to migrate out of the skin epithelium towards the stroma because of their enhanced N-cadherin expression (26). In general, it should not escape our notice that the intermolecular bonding forces of two N-cadherin molecules are nowhere nearly as strong as the E-cadherin homodimer bonding forces. This explains why for instance melanoma cells do not reside in the stroma but are able to cleave their way towards the blood vessels. And there is yet another type of cadherins, the desmosomal cadherin, which also contributes to the structural integrity of epithelia. The downregulation of E-cadherin in carcinomas 

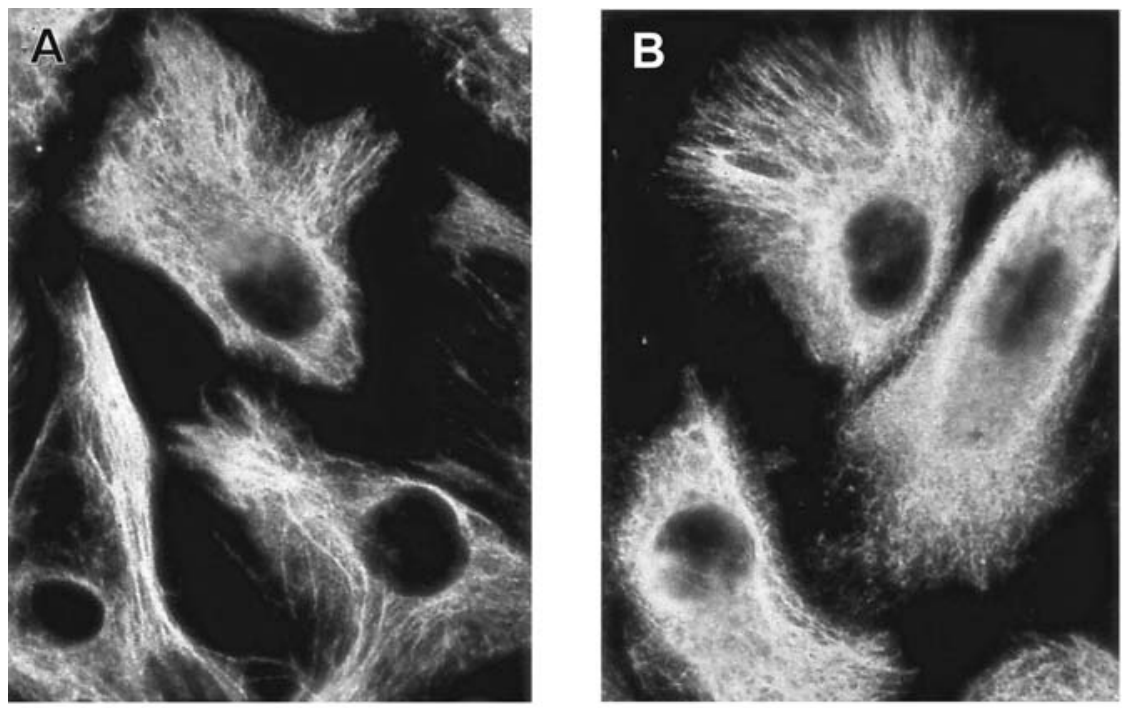

Figure 2. Detection of cytokeratins in renal carcinoma cells by immunofluorescence. (A) Cytokeratin 18. (B) Cytokeratin 19 (magnification x500). Taken from Efferth et al (78) with permission from the International Institute of Anticancer Research, Kapandriti, Attiki, Greece.

is achieved by several distinct mechanisms. For example, frameshift mutations in the E-cadherin gene can lead to a functional loss of the protein which is often observed in breast cancer. Dysregulation can also occur on the transcriptional level. Methylation of the promoter region of the $C D H 1$-gene (E-cadherin gene) is known to be associated with a silencing effect (27). Furthermore, recent publications describe Snail and Slug as transcriptional repressors for the E-cadherin expression (30). Besides mutations and transcription factors, proteoglycans such as MUC-1 can act as steric inhibitors. Finally, germ-line mutations in the $\mathrm{CDHI}$ gene lead to hereditary gastric and colorectal cancer (31). In the course of an EMT not only E-cadherin, but also cytokeratin expression is reduced and the cell polarity gets lost. This represents an important step from the epithelial to the mesenchymal phenotype as those qualities are characteristic for epithelial cells. Cytokeratins are crucial for the shape and stability of the cells and are used as clinical markers. In this context, cytokeratins can be considered as tumor-associated antigens. They can be used either to determine the tissue from which the tumor cells derive or as a tumor marker for the detection of a possible relapse (Fig. 2) (32). The increase of cytokeratins can give evidence for tumor progression and activity even months before clinical symptoms become evident.

Role of $\beta$-catenin within the EMT. The loss of E-cadherin supports invasiveness by attenuation of homotypic cell-cell adhesions (in form of adherens junctions) as described above. Moreover, the resulting loss of the association between cadherins and the cytoskeleton via catenin-linker proteins $(\alpha-$, B-catenin + p120) possibly further supports EMT and invasiveness. The ability to migrate and invade might be increased through special changes in the cytoskeleton of the cells which yield in a rather mesenchymal morphology. $ß$-catenin plays a major role in this context. The basic principle of the B-catenin activity is depicted in Fig. 3.

Normally, a big amount of $B$-catenin is bound to E-cadherin. However, in case the expression of E-cadherin is down- regulated, a lot of free $\beta$-catenin can be found in the cytosol and might subsequently be translocated into the nucleus. Here, it binds to Tcf/LEF transcription factors and consequently induces the expression of genes which promote EMT (33). However, it is still controversial whether the loss of Ecadherin leads to an elevated level of $\beta$-catenin in the nucleus.

In addition, $\beta$-catenin is found to be part of a mitogenic signaling pathway often referred to as the $\mathrm{WNT} / \mathrm{B}$-catenin or canonical pathway. Through this pathway, the cell can respond to Wnt factor signaling. Binding of Wnt proteins to the 'frizzled'-receptor inhibits the activity of the glycogen synthase kinase $3 ß$ (GSK-3ß). In the absence of Wnt proteins GSK-3ß phosphorylates several key proteins and thus marks them for degradation. One of those is B-catenin, which is subsequently ubiquitinated and degraded by the proteasome. In the case of GSK-3ß inhibition, free cytosolic $\beta$-catenin can be translocated into the nucleus. Fig. 3 depicts the Wnt-signaling. The target genes encode special proteins such as cyclin D1 and the Myc oncoprotein, which are important for the EMT as well as cell growth and proliferation. It should be noticed that some members of the Wnt protein family act via other signaling pathways often referred to as $\mathrm{Wnt} / \mathrm{Ca}^{2+}$ (non-canonical pathway) or planar cell polarity pathway (PCP) $(33,34)$.

The E-cadherin gene, $C D H 1$, is thought to be an invasion suppressor gene, because of its ability to inhibit the expression of an invasive phenotype. It was shown in several studies that re-expression of E-cadherin in $\mathrm{CDH} 1$ knock-out cells repressed invasiveness and metastatic potential (26).

EMT induction through stroma signals. As mentioned above, EMT is a critical step in the development of an invasive cancer cell. However, secondary tumors often mirror their ancestors' morphology in the primary epithelial tumor and do not exhibit a mesenchymal phenotype. Hence, it has to be postulated that EMT is reversible.

One of the difficulties in providing evidence for the EMT is the fact that cells in the periphery of the tumor which have just undergone an EMT are hardly distinguishable from other mesenchymal stroma cells. Nevertheless, there are helpful 


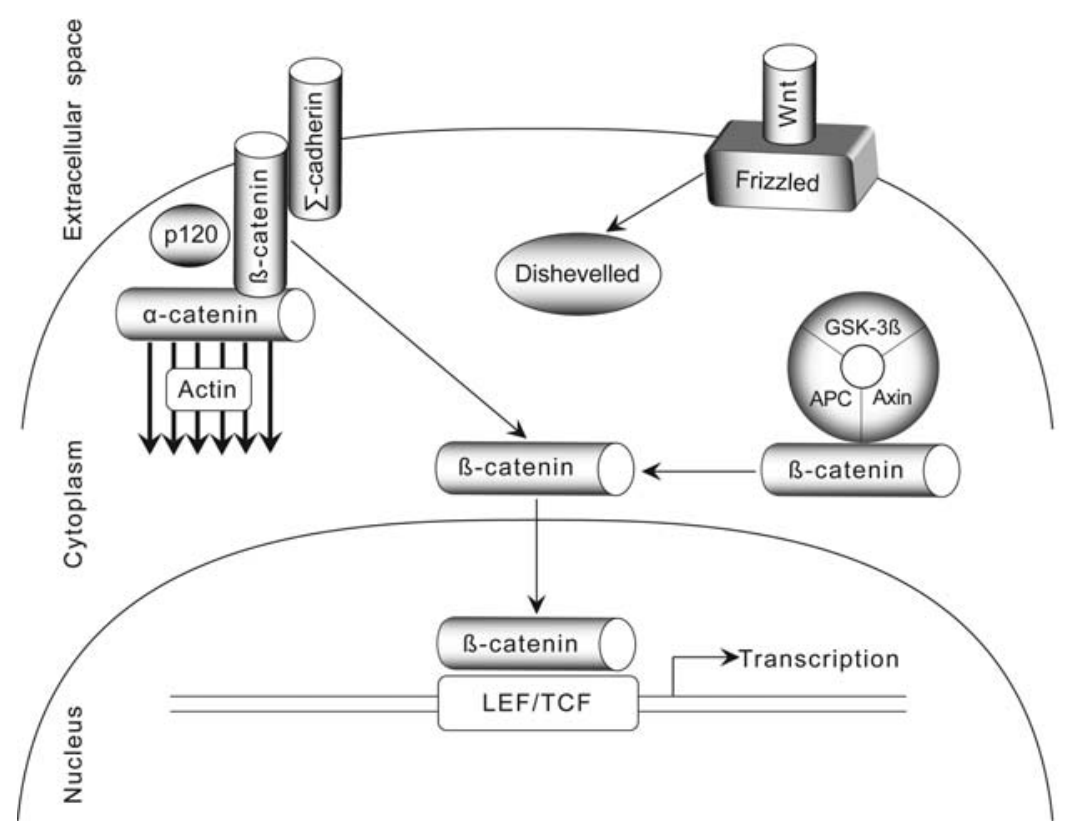

Figure 3. Wnt/ß-catenin signaling pathway.

markers such as fluorescently-labeled antibodies against $\beta$ catenin or the $\alpha v ß 6$-integrin expression.

If cancer cells metastasize and reach another organ, they will not receive the same signal from their new environment. Hence, mesenchymal-epithelial-transition (MET, reversion of EMT) is induced and the original phenotype is adopted. Several factors such as TGF- $\beta$, TNF- $\alpha$, EGF, HGF, IGF-1, E47/e2A, FOXC2, Goosecoid, Snail, Slug, and Twist are known to induce the EMT $(24,35)$.

Transfection of MDCK (Maden-Darby canine kidney) cells with an expression vector containing Twist attenuates the expression of epithelial markers (E-cadherin, B-catenin and $\gamma$-catenin), whereas the expression of mesenchymal markers (fibronectin, vimentin) is increased (36). Twist represents an important transcription factor and a morphogene that regulates cell motility and the organization of organs during early embryogenesis. It was primarily described to be accountable for the formation of the mesoderm in Drosophila research $(36,37)$. In vertebrates it is mainly expressed in the neural crest. Cells undergo an EMT during both formation of the neural crest and gastrulation and are, hence, able to migrate to their new destination point. Thus, it is understandable that Twist affects the EMT. The invasiveness and the metastatic potential of aggressive 4T1 mammary carcinoma cells are reduced by the suppression of Twist. One of the reasons for this finding is the Twist-induced down-regulation of E-cadherin (36) and thus the loss of cell-cell adhesion which yields in increased cell motility. Further studies indicate that Twist is able to inhibit the Myc-induced apoptosis. This factor could be quite relevant since the loss of cell-cell adhesion normally leads to apoptosis (37). In addition, Twist expression was found in highly aggressive melanomas and can be used as a prognostic marker for poor outcome in this case. A similar situation was found in particularly aggressive neuroblastomas (37). Neuroblastoma and melanoma cells are derived from the neural crest where Twist is normally active. Accordingly, the expression of Twist in those cells should not come as a surprise. The precise mechanisms by which Twist is reactivated to induce EMT are unknown.

As stated before, several other mechanisms can influence EMT. Some of them (e.g., Twist, Snail and Slug) are localized at the same place in the embryo during early embryogenesis and consequently it is likely that they work synergistically (37).

Other factors such as TGF- $\beta$ (transforming growth factor $\beta$, a cytokine) are able to cooperate with mutated alleles of oncogenes such as Ras. It has been shown that Ras-transformed MECs (mouse mammary epithelial cells), which had been exposed to TGF- $\beta$, displayed a loss of epithelial and an acquisition of mesenchymal markers, i.e., they underwent EMT. After those cells had performed EMT, they produced their own TGF- $\beta 1$ which could thus operate via autocrine signaling and enabled the cells to keep their mesenchymal phenotype during migration (26). The PI3K (phosphatidylinositol-3-kinase, a downstream-target of Ras) has a central role in this process since it attenuates the cytostatic effects of TGF- $\beta$. Moreover, it is known that TGF- $\beta$ as well as TNF- $\alpha$ (tumor necrosis factor $\alpha$ ) stimulate the NF- $\kappa \mathrm{B}$-signaling (nuclear factor $\kappa \mathrm{B}$ ). Besides its impact on immune response, proliferation and apoptosis, the NF-кB-pathway seems to be crucial for EMT since its inhibition shuts down the EMT (38). Cells of the innate immunity such as macrophages are also of vital importance in the context of metastasis. Macrophages produce TNF- $\alpha$ in large amounts and there is evidence that they also produce most of the EGF in the case of breast cancer. The expression of CSF-1 (colony stimulating factor) by the cancer cells recruits tumor associated macrophages (TAMs) which in turn produce EGF and thus the cells reciprocally stimulate each other. These cancer cells express only EGF-receptors whereas TAMs exhibit only CSF-1 receptors (39). Activation of the EGF-signaling in cancer cells causes them to secret CSF-1 and promotes both motility and invasiveness. If cancer cells in the murine model are not capable of producing CSF-1, the typical activation of 


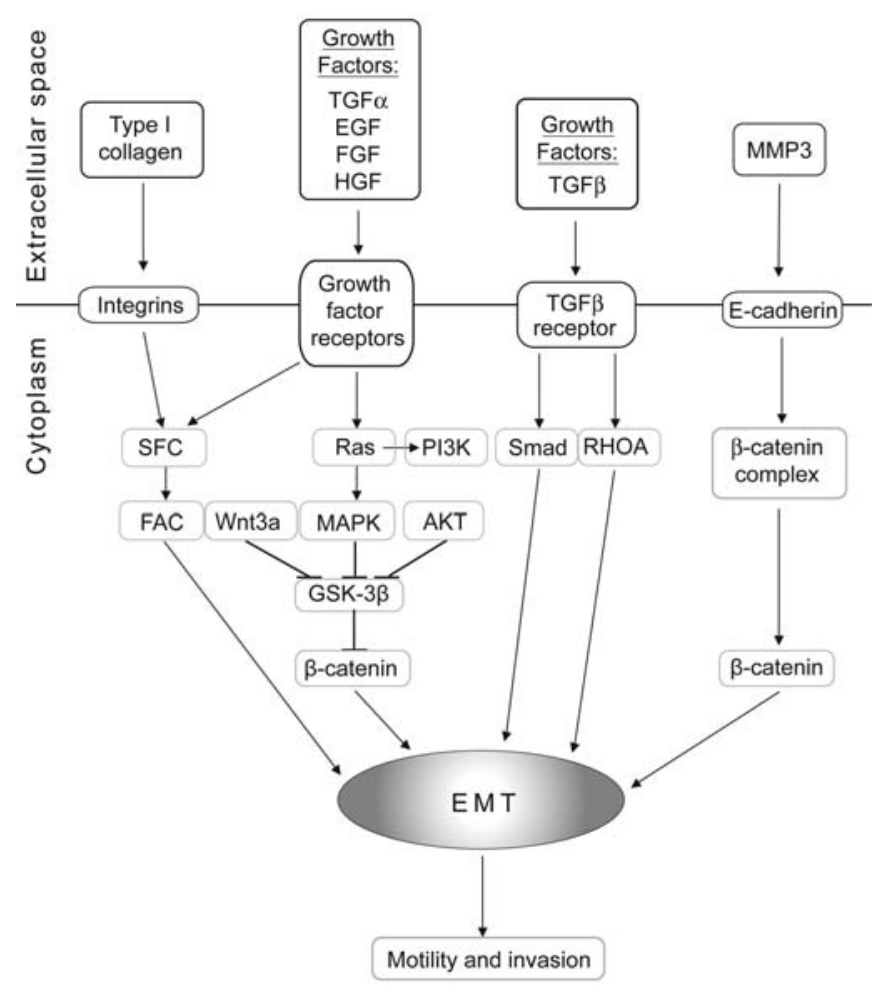

Figure 4. Factors influencing epithelial mesenchymal transition (EMT).

tumor associated macrophages (TAMs) and thus the intensive expression of EGF is missing (40). Studies on breast carcinomas have shown that the absence of CSF-1 and thus TAMs do not inhibit the proliferation of the primary tumor, but it prevents lung metastasis (41). In other words, a mechanism describing the promoting effects of stroma macrophages on the invasiveness of cancer cells has been uncovered. The invasion-supporting role of EGF in carcinogenesis was verified in several studies (42). The detailed mechanisms remain obscure, but it is known that EGF activates Ras as well as dephosphorylates and down-regulates FAK (focal adhesion kinase) (42). It should be noted that a variety of transcription factors, cytokines, stroma cells and signaling pathways influence the EMT. A simplified scheme of the factors is displayed in Fig. 4.

Impact of CD44 on the process of metastasis. CD44 proteins are adhesion molecules which can be found on the surface of various cells. They are a class of transmembrane glycoproteins which are able to bind to several molecules and play a vital role in cell-cell adhesion, migration and lymphocyte homing (43). CD44 primarily acts as a receptor for hyaluronic acid (hyaluronan), a glycosaminoglycan and integral part of the ECM. Besides hyaluronic acid, CD44 also binds to other molecules such as collagen, osteopontin, laminin, MMPs (matrix metalloproteinases), fibronectin, chondroitin sulphate and heparan sulphate $(43,44)$. The CD44 gene contains 20 exons, 10 of which are variable and inserted by alternative splicing. The composition of CD44 is illustrated in Fig. 5. The ubiquitous occurring variant of CD44 contains none of those variable exons, whereas several splicing isoforms can

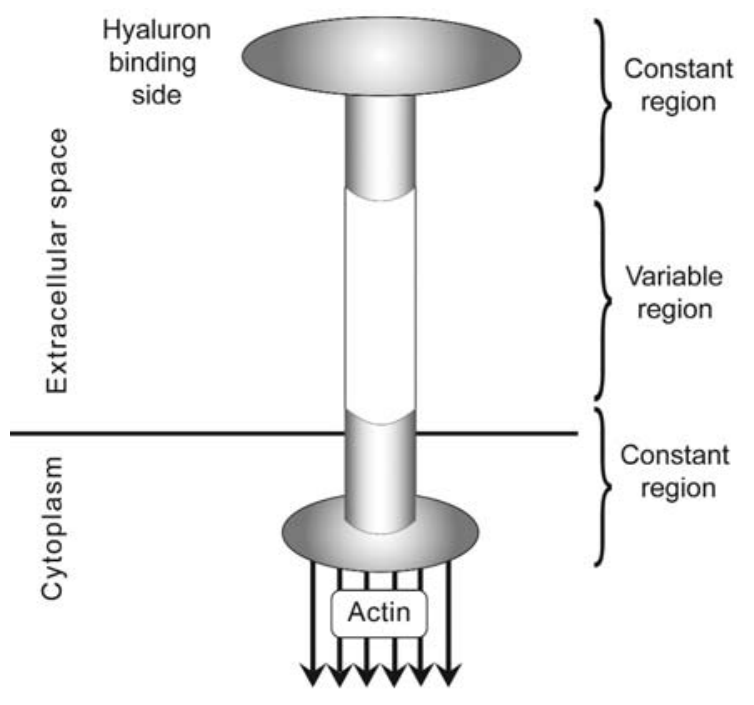

Figure 5. Structure of CD44.

be found in proliferating epithelial cells as well as in activated lymphocytes. Furthermore, post-translational modifications such as glycosylation can influence CD44's binding affinity to hyaluronic acid.

Via ankyrin and other proteins (ezrin-moesin-radixin family) the cytoplasmic domain of CD44 interacts with the actin cytoskeleton (43). Little is known about the intracellular changes which occur after the binding of a ligand or after splitting off several protein domains. However, it is assumed that rearrangement of the cytoskeleton promotes migration and invasion. In addition, several splicing-isoforms of CD44, particularly those containing the V6-exon, were found to be present in several neoplasias. An etiological connection between the presence of CD $44 \mathrm{v}^{+}$isoforms and the ability to metastasize was detected in the murine model (43). It has been shown by RT-PCR and Southern blotting that human colon- and mammary carcinomas usually involve large CD44 splicing isoforms. However, it should be mentioned that some healthy adenocytes are also CD44v6 $6^{+}$. The presence, respectively the over-expression of distinct isoforms can be used as a marker for cancer development or aggressiveness $(43,45)$. CD44 also acts via several signaling pathways. For example, the over-expression of CD44v6 together with c-Met, which is the receptor for HGF (hepatocyte growth factor), is frequently observed. c-Met is a common receptor tyrosine kinase with stimulating effects on migration, invasion, proliferation and EMT. There is evidence that expression of CD44v6 is crucial for the activation of c-Met by HGF (27). The extracellular domain is essential for the HGF-induced auto-phosphorylation of c-Met, whereas the cytoplasmatic tail of CD44v6 is vital for signal transduction to downstream effectors such as MEK or MAPK (27). Activation of those pathways leads to proliferation, migration and evasion of apoptosis. Similar phenomena have been described for other isoforms. For example, CD44v3 facilitates the interaction of HB-EGF (heparin-binding EGF-like growth factor) with its receptor-tyrosine-kinase ErbB4, which belongs to the EGFR family. CD44v3 probably activates MMP-7 which proteolytically converts HB-EGF into the active, receptor-binding form. ErbB4 is an oncogene and activation of its protein 
product once more promotes tumorigenesis by stimulating several signaling pathways (RAS/MAPK, Akt/PKB) (27). Despite the already mentioned interactions, separated fragments such as sCD44 or CD44ICD are relevant for CD44s' impact on invasion and metastasis. sCD44 is split off by MMPs, can be detected in the blood and other bodily fluids and acts as antagonist and regulator. The cytosolic fragment CD44ICD is translocated into the nucleus and operates as a transcription factor.

In conclusion, CD44 has a major impact on the development of an invasive phenotype by inducing changes in the actin cytoskeleton, different splicing-isoforms and through CD44 fragments.

Extracellular proteases. The cells of a tumor have to acquire the capability to pave the way through the extracellular matrix and several tissue layers in order to invade and metastasize. Therefore, they must activate proteolytic enzymes which degrade the ECM. In this process, not only proteins, but other components of the ECM such as glycosaminoglycans are degraded. Probably the most important effectors in this process are the zinc-dependent, in tumors frequently overexpressed matrix metalloproteinases (MMPs). Most of these endopeptidases are secreted by stroma cells such as macrophages, mast cells and fibroblasts. The cellular substrates of MMP degradation are manifold and include fibronectin, collagen, laminin and proteoglycans. Soluble MMPs are always secreted as inactive pro-enzymes into the ECM and must therefore be activated by other enzymes (26).

Besides the proteolysis, MMPs also affect tumor neoangiogenesis and proliferation by activating/deactivating several growth factors. The so-called TIMPs (tissue inhibitors of metalloproteinases) play an important role in the complex regulation of MMPs. They inhibit the MMPs and, thus, the balance between TIMPs and MMPs is of vital relevance for invasion and metastasis. Nowadays, $>20$ distinct MMPs are known: collagenases (MMP-1/8/13/18), gelatinases (MMP-2/9), stromalysines (MMP-3/10/11), matrilysines (MMP-7/26), membrane-bound MMPs (MMP-14/15/16/ 17/24/25) and other MMPs, which do not fit into the listed groups (46). The function of collagenases is the degradation of triple-helical collagen fibers, gelatinases digest denatured collagen and gelatins, stromelysins activate pro-MMPs through proteolytic cleavage and digest several other components of the ECM, matrilysins process cell surface molecules such as CD95-ligand (CD178) and E-cadherin, membrane-bound MMPs activate pro-MMP-2 and other ECM-molecules. However, MMPs should not be considered simple effectors of the cell - the dysregulation of MMPs is capable of promoting the conversion of a formerly healthy cell into a malignant tumor cell.

So far, MMP inhibition in tumor therapy takes place on three levels: transcription, pro-MMP activation and MMP inhibition. The blockage of transcription was achieved by interferons and small molecules which inhibit MAPK- or ERK-signaling. In addition, inhibitors of transcription factors such as AP-1 and NF- $\mathrm{B}$ were used. Since those approaches affect various genes, antisense-oligonucleotides or RNAi (RNA-interference) might be more appropriate. Inhibition of MMP-activation can be facilitated by antibodies or small molecules against membrane-bound MMPs. Furthermore, protease inhibitors (as used in HIV-therapy) are capable of restraining MMP-activation. The usage of TIMPs has proven to be inappropriate due to intense side effects. A direct inhibition of MMPs can be accomplished by pseudomimetica (batimastat, marimastat) which imitate the active center of MMP-substrates and chelate MMPs' zinc ion with a hydroxamate group. Yet, the hitherto existing results are not very encouraging (47). The unresolved problems are often due to the manifold physiological MMP functions.

Another proteolytic enzyme called uPA (urokinase plasminogen activator) is crucial for MMP regulation. UPA is secreted by stroma cells and binds to its own receptor (UPAR) on the cell surface. That way, the protease becomes active and cleaves extracellular substrates in its surrounding area. For example, uPA cleaves plasminogen into plasmin which in turn activates MMP-1, 2, 3, 9, and 14 as well as TGF- 11 (48). The inhibition of the uPA-uPAR-system could attenuate tumor growth and metastasis in vivo. Besides MMPs, there are many other proteases in human soma cells, which are encoded by hundreds of different genes. They are classified as serine-, threonine-, cysteine-, aspartate- and glutamateproteases. Only few of them have been sufficiently studied and it seems likely that a substantial part of them is of importance in invasion and metastasis of cancer cells. The individual steps of the uPA-uPAR-system are summarized in Fig. 6.

Cell motility. As discussed in the previous sections, several extracellular proteases degrade the ECM and, hence, establish a basis for the invasion of tumor cells. However, what is known about the exclusive activity of those proteases is not sufficient for a comprehensive understanding of cell motility. In culture, cell motility can be achieved by application of several growth factors. Hepatocyte growth factor (HGF) for example is a potent inducer of motility in epithelial cells. The receptor for HGF is MET (mesenchymal epithelial transition factor) which is expressed by epithelial cells. In analogy, EGF is capable of inducing motility in mammary carcinoma cells. The macromolecular reasons for the acquisition of cell motility are the remodeling of the actin-cytoskeleton, as well as the formation and dissolving of adhesion complexes.

The cell develops lamellipodia (flat, wide membrane rags) which stretch in the moving direction. On the front end, integrins build new focal contacts between ECM and lamellipodium, while the connections on the end are dissolved. In this manner the cell moves and continuously secretes the above mentioned proteases. On the edge of a lamellipodium one can detect spiky structures, the filopodia, which are also formed by the reorganization of actin fibers. The exact function of filopodia has not been determined yet, but it is possible that they act as sensing devices (26).

The organization of cell shape as well as motility is subject to supervision of the protein family of Rho GTPases. Rho proteins are active when GTP is bound and inactive when GDP is bound. There are three groups of Rho proteins with $>20$ members altogether: the actual Rho proteins, the Rac proteins and $\mathrm{Cdc} 42$. Rho proteins are lipoproteins which carry a lipid part on their $\mathrm{C}$-terminus that allows the association with biomembranes. It should be noted that the process of cell motility is dependent on the localized activation of those 


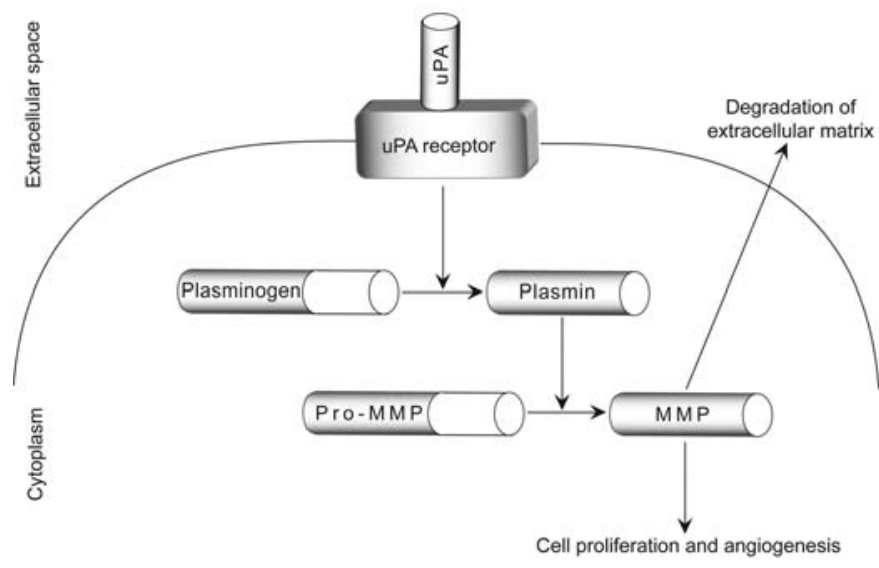

Figure 6. The uPAR system.

differently operating proteins. Only in this manner a directed movement is possible. The activation of special receptor tyrosine kinases (RTKs) by growth factors ultimately results in the activation of many Rho proteins. For instance, the application of PDGF (platelet-derived growth factor) to fibroblasts leads to enhanced cell motility (26).

Through the activation of PDGF receptor, several downstream effectors such as Raf, Ral-GEF and PI3K (phosphatidylinositol-3-kinase) are stimulated via Ras. PI3K is of substantial relevance for cell motility because it ultimately activates some members of the Rho-family of G-proteins via specific GEFs (guanine nucleotide exchange factors). Rac and Cdc42 in their part seem to induce the expression of certain secretion-proteases (particularly MMPs). Rho proteins also control the contraction of the cell body. Those contractions pull the rear part of the cell in moving direction. The formation of actin bundles (stress fibers) in the cytoplasm is enhanced during this process. Until now, more than 20 different GEFs of the Rho protein family have been discovered, which illustrates the complexity of regulation. $\mathrm{Cdc} 42$ is capable of inducing filopodia and also conveys stimulating effects on cell motility (49).

In conclusion, the members of the Rho family (Rho, Rac and Cdc42) play an important role in the regulation of cell motility and thus invasion and metastasis. However, the detailed molecular principles are not understood to a sufficient degree yet.

Chemokines. Chemokines are rather small (8-14 kDa), chemotactic cytokines which determine the direction of migration of various cells such as leukocytes and cancer cells. They bind to G-protein linked receptors (50) and are divided into the following four subgroups: CXCL, CCL, CX3CL and CL (46). By now, $>50$ chemokines and 18 chemokine receptors are known. The receptors possess seven hydrophobic transmembrane domains and belong to the rhodopsin super-family. The N-terminus is located outside the cell, the C-terminus with its threonine and serine phosphorylation sites in the cytoplasm. The intra- and extra-cellular receptor domain possesses three loops in its structure (50). One can distinguish between inflammatory and homeostatic chemokines, the latter being constitutively expressed. Inflammatory chemokines (e.g., CCL3, CCL5, CXCL10) are released in consequence of special stimuli (injuries, inflammation) and induce leukocyte homing to the affected tissue site. Homeostatic chemokines are involved in the development and the homeostasis of the immune system. As a third group, the 'dual function' chemokines have to be mentioned. They are released during inflammation and recruit lymphocytes in the course of the immune response. Binding of chemokines to their receptors activates specific G-protein linked signal transduction pathways (phospholipase-C- $\beta$ isoforms, phosphatidylinositol3-kinases, several Src-kinases) (50). In the context of cancer, chemokines represent important direction signs of invasion and metastasis. There are special chemokine receptors which enhance the organ-specific metastasis and, thus, increase the 'homing factor'. An important representative of this group is CXCR4 which occurs on the surface of malignant breast cancer cells. The ligands of this receptor can be found on the surface of liver-, lung- or bone cells. Therefore, an increased amount of metastases in these organs can be observed. In addition, CXCR4 was shown to be vital for the lymph node specific metastasis (CXCL12/SDF-1-ligands) of melanomas and mammary carcinomas $(51,52)$. Moreover, chemokines can operate as paracrine/autocrine growth factors, as survival signals or they can influence angiogenic/angiostatic processes and tumor-specific immune responses.

As stated above, chemokines regulate tumorigenesis in at least four different ways: By modulation of angiogenesis (53), by activating the tumor-specific immune response (54), by acting as homing factors (52), as well as by autocrine growth stimulation (55). An antibody-based depletion of chemokines could be a new therapeutic approach. Accordingly, it has been shown that $\alpha$-IL-8 (angiogenic chemokine) monoclonal antibodies significantly inhibited tumor vascularization and growth in SCID mice with NSCLC (non-small cell lung cancer) (53).

Intravasation. The term intravasation refers to the process in which a cancer cell enters the lumen of lymphatic or blood vessels. This crucial step of metastasis is based on the penetration of endothelial cells by the cancer cells, as well as the previous tumor angiogenesis. Thus, angiogenesis promotes the tumor growth in two distinct ways: by providing the necessary nutrients (and the evacuation of metabolic waste products), as well as by the direct access of neoplastic cells 
to the blood vessels, which are vital for metastasis. Not only the blood system, but also the lymphatic system plays an important, even though less obvious role. The lymphatic system collects the interstitial fluid and conveys it via the lymph nodes and lymphatic vessels back again into the (blood) circulation. Thus, cancer cells can either directly or indirectly (via lymphatic vessels) reach the circulation provided that they do not persist in the next lymph node. The first metastases are often found in the lymph nodes which, therefore, are of great importance for tumor staging and prognosis (51). The biggest human lymph is called ductus thoracicus, which disembogues in the left venous angle (confluence of vena subclavia and vena jugularis interna). Some researchers claim that carcinomas often spread initially via the lymphogenous system, whereas sarcomas tend to spread via the hematological system (51). The penetration of lymphatic vessels seems to be easier due to the absence of a continuous basement membrane and a coating layer of pericytes, as well as their weak inter-endothelial connections (51).

At the border of tumor and stroma there are often enlarged lymphatic vessels whose formation seems to be a consequence of the VEGF-C expression that drives lymphangiogenesis. Tumors with enhanced VEGF-C expression exhibit an increased metastatic potential especially for the draining lymph nodes (26). Lymphatic vessels rarely occur in the midst of a solid tumor mass, which is an explanation for the increased pressure rates in solid tumors.

In order to penetrate the vessels, several proteases are secreted, which degrade the endothelium and enable the cells to enter the lumen of the vessel. The migration to the vessels is facilitated with the help of several chemokines as well as with the altered expression of several adhesion molecules (such as $\mathrm{N}$-cadherin). The term extravasation refers to the reciprocal process of leaving a vessel and infiltrating the surrounding tissue, which is discussed in detail in the section Extravasation.

Circulation. The third step of the metastatic cascade is the circulation, that is to say the migration of a cancer cell in the lumen of lymphatic or blood vessels to a distant tissue site. The circulation in the blood is another critical step of metastasis due to the predominant conditions which can be toxic for tumor cells. There are various reasons for this: Normally, most cells can not survive and proliferate without adhesion onto other cells or solid substrate. Cancer cells, however, have to evade this restriction in order to build secondary tumors. This stage is called 'anchorage independence'. After a certain time, normal cells activate a special form of programmed cell death (PCD) called anoikis in case they can not adhere to solid substrate or other cells. If a cancer cell is still anchorage-dependent at the time it enters circulation, anoikis is activated and the cell dies (56). In addition, if cancer cells are not yet independent of exogenous growth factors which are secreted by stroma cells, apoptosis might be induced (due to the absence of those growth factors in the circulation). Particularly in smaller vessels such as the arterioles or capillaries high shear forces, which damage the cells, can occur. Those forces are likely to be one of the major reasons for the metastatic inefficiency.
Finally, the high concentration of oxygen and lymphocytes can impair the tumor cells. Because of the presence of those adverse conditions a selection of particularly aggressive and resistant cancer cells takes place (46).

Neoplastic cells display a technique to antagonize these toxic conditions: They form micro-emboli (syn. microthrombi, thrombo-emboli). These are agglutinations of tumor cells with thrombocytes and erythrocytes. The formation of those localized micro-emboli is mainly due to the action of the 'tissue factor', which is a protein strongly expressed on malignant carcinoma cells but not that much on benign tumor cells and healthy epithelial cells. The tissue factor interacts with special proteins in the plasma to initiate the clumpingcascade. In this process thrombin is activated, which converts fibrinogen into fibrin and thus keeps the clumping together (26). The importance of micro-thrombi was analyzed in knock-out mice which lacked specific components of the clumping-cascade (e.g., fibrinogen, platelets). Melanoma cells were administered intravenously into the knock-out mice and the metastatic efficiency was verified. In comparison to the control group, metastasis was decreased by $>90 \%$ (57). In therapeutic work, circulating tumor cells are also used as a marker or a prognostic factor, respectively. It should not come as a surprise that current studies detected a significant correlation between the presence of circulating cancer cell in the peripheral blood and a poor prognosis (51).

Extravasation. If cancer cells survive the adverse conditions in the vessels and reach the bigger venous blood vessels, they are carried further by the blood stream and reach, after the passage of the heart, the capillary network of the lung. At that point, there is a high probability that the cancer cells get entangled in the capillary system of the lungs. This can be explained by the size of the cancer cells $(\sim 20 \mu$ m diameter $)$ in relation to the capillaries ( $\sim 3-8 \mu$ m diameter) (26). Furthermore, cancer cells, unlike erythrocytes, are not very elastic and often form micro-thrombi. Thus, chances are high that they become stuck in the arterioles.

Another theory indicates that cancer cells use specific cell surface receptors (such as integrins) to adhere to the endothelium of blood vessels of specific organs (58). Various studies have found that several types of cancer frequently spread metastases in the same organs. This is due to a capillary network downstream of the metastases and is referred to as 'hemodynamic factor'. However, the situation seems to be more complex. Several molecules such as the CXCR4receptor, which plays a vital role in organ-specific metastasis, have been discovered and are also referred to as 'homing factors'.

However, not all cancer cells get stuck in the lung capillaries. Two theories offer explanations for their escape. The first one assumes that the cells discard a great amount of their cytoplasm in order to form smaller, but still vital cells, which can passage the lung capillaries. The other holds that cancer cells can avoid the capillary network by using 'arterial-venous shunts' (direct connections which bypass the capillaries). When the cancer cells leave the lung capillaries and reach the general arterial vessels, they can migrate into various kinds of body tissue. The now occurring extravasation can take place in two different ways. One possibility is that cancer 
cells start to proliferate in the lumen of a vessel. Due to the growth of the tumor, the vessel wall is destroyed and thus the cancer cell's way into the tissue of the organ is paved.

The second possibility for the cancer cells to penetrate an organ is similar to the intravasation and includes the degradation of endothelium and basement membrane through proteolysis. Leukocytes (e.g., monocytes or neutrophil granulocytes) also have to extravasate in great quantity and over a short period of time out of the blood vessels into the tissue during an infection. In the course of this process which is called 'diapedesis', leukocytes adhere via selectins to the endothelium and perform the so-called rolling adhesion to finally interact with endothelial cells, which in turn enable the passage of the leukocytes. Various molecules such as LFA-1 + ICAM-2, CD3 + ICAM-1, as well as PECAM (CD31) (59) are involved. The complete process takes place in less than a minute.

Even though older literature describes the extravasation of leukocytes and tumor cells as being quite similar, metastasizing cancer cells often lack the capabilities and receptors required to that end and are thus reliant on the above-mentioned techniques.

Colonization, proliferation, and angiogenesis. The colonization, subsequent proliferation and the process of angiogenesis together represent the final step of the invasionmetastasis-cascade and contribute to the already mentioned metastatic inefficiency. The new environment, the so-called 'micro-environment', does not provide the same survivaland growth factors as the original tissue, which enabled the tumor to proliferate (26). Without those special, physiological factors many metastasizing cells die or reside without proliferation as micro-metastases. Therefore, the amount of senescent micro-metastases in cancer patients usually exceeds the amount of micro-metastases that grow to a detectable size. Micrometastases can be tracked down through histopathological methods such as antibody-based cytokeratin staining in blood and bones or $\alpha$-EpCAM antibodies (epithelial cell adhesion molecule) in the case of lymphatic micro-metastases.

The detection of cytokeratin positive and hence epithelial cells in completely mesenchymal tissues provides evidence for micro-metastases. This technique makes it possible to track down single cell micro-metastases amongst $10^{5}$ or even $10^{6}$ surrounding mesenchymal cells. It is assumed that EMT (discussed in Epithelial mesenchymal transition and modified gene expression of cadherins and cytokeratins.), which takes place at the beginning of the invasion, is reversed during colonization. As stated above, this process is called MET (mesenchymal-epithelial-transition) and enables the tumor cells to adopt their original epithelial character.

The great amount of micro-metastases that is frequently found in the body of a patient also can be seen in the context of genetic evolution. The cells experience various mutations in the course of time which often give them new phenotypic qualities. However, those mutations rarely enable the cancer cells to proliferate and form a clinically relevant secondary tumor, for instance through the achievement of independence from mitogenic factors. If that is the case, the relevant clone is now able to proliferate and it is possible that cells decoct from the tumor to build secondary metastases. In this case the patient faces a poor prognosis. Due to their distinctive capillary network, lung, bones, brain, liver and adrenal glands are frequently affected by metastases.

After the colonization and first proliferation of the secondary tumors, the neoangiogenesis takes place. Neoangiogenesis is the formation of new blood vessels in the direction of a neoplasia to provide it with nutrients and to remove metabolic waste products. As mentioned above, a tumor without vascularization is only able to grow to a size of 1-2 mm. The stadium of tumor growth prior to angiogenesis is referred to as avascular phase. Not until entering the vascular phase, a tumor is able to proliferate exponentially. The balance of pro- and anti-angiogenic factors regulates the transition from the avascular to the vascular phase. This process is called 'angiogenic switch'. For example, angiogenic oncoproteins such as Ras can operate as angiogenic factors and enhance the expression of pro-angiogenic proteins. Those include VEGFs, FGF (fibroblast growth factors), PDGF, and EGF. In addition, the expression of angiogenesis inhibitors such as angiostatin or thrombospondin can be down-regulated by oncogenes (46). Mutations of TP53 can also affect the angiogenic balance, because they reduce the expression of thrombospondin and increase the VEGFA expression. The hypoxia prevalent in a tumor also induces via HIF-1 (hypoxiainducing factor 1) the increased secretion of VEGF (operates as a chemokine), PDGF, Glut-1, and Glut-3 (glucose transporter) $(60,61)$. In addition, fibroblasts are provoked to produce pro-angiogenic substances. The vascularization of a tumor differs strikingly in its structure from normal vessel networks: it is more chaotic, often enlarged, contains dead ends, the blood flow is slower or oscillates, and sometimes there are holes which can cause hemorrhage.

The hitherto existing angiogenesis inhibitors suppress either proliferation of the endothelial cells or they induce them to undergo apoptosis. Recent studies also deal with angiogenic gene therapy in which adenoviral vectors are used for the transgenic expression of e.g., endostatin. Endostatin inhibits (as a cleavage product of collagen XVIII) the proliferation of the endothelium. In these studies tumor growth and metastasis could be decreased in vivo (62).

Metastasis suppressor genes. In the early 1990s, the first metastasis suppressor gene called nm23 (non-metastatic 23) was identified, which provided the functional proof for the existence of genes specifically regulating metastasis (63). These genes inhibit metastasis without affecting the primary tumor proliferation. Another example is maspin, which binds to collagen and thus fosters tissue cohesion. Breast cancer cells with a decreased expression of maspin, therefore, tend to metastasize. Conversely, the metastasis of breast cancer cells was inhibited in vivo by maspin (64).

Nowadays, genes which allow the spread of cancer cells to distant tissue sites, but inhibit the colonization of these micro-metastases are also referred to as metastasis suppressor genes. Examples are: KISS1, JNKK1/MKK4, MKK6, MKK7, TXNIP, $n m 23-H 1$ or SSeCKS (65). Those genes often affect fundamental signaling pathways of the cell, such as the MAPK/ERK- (mitogen activated protein kinase/extracellular signal regulated kinase) or the SAPK/JNK- (stress-activated protein kinase/c-Jun N-terminal kinase), as well as the p38- 
pathway. Furthermore, proteins of the Rho super-family (Rho, Rac, Cdc42) operate as metastasis suppressor proteins.

Monoclonality and cancer stem cell theory: do all cancer cells share the same abilities? In the following chapter, the terms monoclonality, cancer stem cell theory and its implications, as well as the question whether all cells of a tumor share the same capabilities, shall be discussed.

The principle of monoclonality in tumor biology. When tracing the development of a tumor down to its origin, the question of how many cells it has originally been derived from emerges. In principle, there are two different possibilities which should be taken into consideration: either all cells of a tumor could be derived from a single cell (monoclonality), or the tumor could be a heterogeneous mass derived from multiple cells (polyclonality). In order to answer this question, the cells could be genetically analyzed. If a tumor cell exhibits a rare and highly specific mutation which can be accurately localized, the other cells of a tumor could be checked at random for this mutation. If all cells exhibited the same, rare mutation, this would provide evidence for the monoclonality of the tumor (26). Indeed, specific genetic or chromosomal changes such as the randomly proceeding inactivation of a specific enzyme in the Barr body in heterozygous, female individuals were used to answer this question. The enzyme was the glucose-6-phosphate-dehydrogenase. $30 \%$ of Afro-American women are heterozygous for this locus. This and other experimental findings provided evidence that virtually all tumors are of monoclonal origin (66). How-ever, there are other researchers who regard those results as insignificant and still question the theory of tumor mono-clonality (67).

Cancer stem cell theory. Stem cells are pluripotent cells with a high replicative potential, which can renew themselves and are able to differentiate into different cell types via progenitor cells. There are three main types of stem cells: i) Embryonic stem cells (ES-cells) which are derived from the embryoblast i.e., the inner cell mass of a blastocyste from which the embryo develops itself. ii) Embryonic germ cells (EG-cells), which can be isolated from primordial germ-line cells from fetuses. iii) Adult/somatic stem cells which can be found in various tissues (bone marrow, brain, blood, liver, retina, etc.). In classical tumor therapy of the last decades, adult, hematopoietic stem cells (HSCs) were used to reconstitute the hematopoietic system or to induce 'graftversus-leukemia-effects' after myeloablative chemo- or radiotherapy (68). More recent studies are aimed at using stem cells as transport vehicles for tumor cell specific application of proliferation-inhibiting and pro-apoptotic substances. This is based on the fact that tumors secret chemokines such as VEGF, recruit pericytes for angiogenesis and encourage MSCs (mesenchymal stem cells) to build the tumor-supporting stroma. The goal is to transfect MSCs by using adenoviral vectors, which induce the intense expression of e.g., interferon- $\beta$ and thus specifically harm the tumor (69).

During recent years, a new theory of the development and proliferation of cancer cells was intensely discussed among experts: the cancer stem cell theory (70). This theory indicates that a solid tumor is heterogeneous and consists of two types of cells: normal cancer cells that rapidly, but

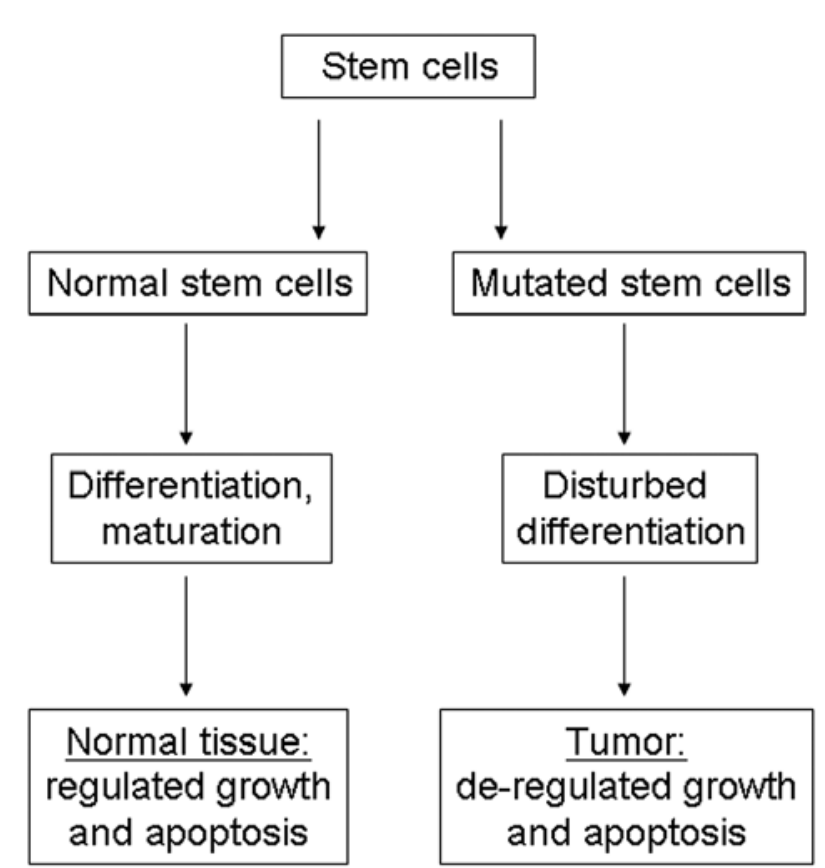

Figure 7. Development of cancer stem cells.

not infinitely, proliferate (transit-amplifying cells) and the 'cancer stem cells' which exhibit an indefinite proliferation capacity and are able to build clinically relevant secondary tumors (71).

The hitherto existing notion indicated that all tumor cells are more or less equal and have a similar metastatic potential, the latter was thought to be quite limited due to several factors (immune system, toxic environment in the blood, altered microenvironment, etc.). The new theory regards a tumor as an aberrant organ which consists of stem cells and differentiated cells - in analogy to the hematopoietic system, for instance. The question of the origin of cancer stem cells is still to be discovered, yet there are two theories. Due to the fact that several mutations are required to transform a normal cell into a cancer cell and stem cells have to shed all genetic proliferation limitations, stem cells are probably derived from either normal adult stem cells or from progenitor cells, which have acquired the ability of self-renewal (69) (Fig. 7).

Astonishing experiments as the one described below support this hypothesis: tumor cells were separated on the basis of surface markers (e.g., CD44, CD24, CD133, Lin) by FACS (fluorescent activated cell sorting) technology $(72,73)$. Afterwards, the different fractions were injected into NOD/ SCID-mice in different amounts with intriguing outcome: even 100,000 cells of one fraction could not establish a solid tumor in the mouse, whereas only 100 cells of the other fraction resulted in cancer $(71,73)$. This new model is able to explain why only some specific micro-metastases actually build clinically relevant tumors (most micro-metastases do not contain cancer stem cells). Furthermore, the effect of several drugs such as the 'small molecule' Gleevec (targeted at several tyrosine kinases such as the Abl-kinase in chronic myelogenous leukemia or c-Kit (74) could be explained. Gleevec can shrink the cancer below the clinical detection limit. However, if the patient stops taking Gleevec the cancer 
reoccurs. This might be due to the fact that Gleevec kills most of the proliferating, transit-amplifying tumor cells but not the crucial cancer stem cells. The latter are, to a great extent, not part of the active cell cycle and are, thus, largely unaffected by Gleevec (and most other current cancer therapeutics). Furthermore, some scientists believe that cancer stem cells and their genetic instability are of great importance for the development of drug resistances (75). It was detected that they express high levels of ABC (ATP-binding cassette) drug transporters, which are known to be highly important for tumor resistance, have increased levels of DNA repair and seem to be less capable to undergo apoptosis (76).

The cancer stem cell theory has extensive consequences for our understanding of tumor biology as well as for new therapy approaches. Most current chemotherapeutics aim at killing strongly proliferating cells. Yet, it has to be considered that stem cells - cancer stem cells as well as other adult stem cells - divide only once in a while to either renew themselves or finally build differentiated cells. The differentiated cells on their part are able to proliferate rapidly and are therefore well antagonized by current chemotherapeutics. However, this does not apply to cancer stem cells. The latter can play a role in tumors in three different ways: i) Mutations of adult stem cells or progenitor cells can yield to the formation of the primary tumor. ii) During chemotherapy most cancer cells are destroyed. However, if not all cancer stem cells are destroyed, the patient will face a relapse. iii) Cancer stem cells migrate to distant tissue sites and build clinically relevant metastases (69). Through the effect of a continuous chemotherapy a tumor could develop which contains virtually only cancer stem cells. The intent is to identify specific cancer stem cell markers (in analogy to Thy-1, CD34 and c-Kit in HSCs) and to develop drugs which also eliminate cancer stem cells (69). In this manner, a patient could even be cured, because the normal cancer cells which are left exhibit only a finite proliferation capacity. However, elimination of cancer stem cells seems to be difficult due to the fact that they do not apparently exhibit active hyper-proliferation signals such as tyrosine kinases (69). Even though, there are already drugs which also affect cancer stem cells. One example is rituximab, an anti-CD20 monoclonal antibody that is used against B-cell tumors. Anti-cancer stem cell drugs need time to take effect due to the fact that they only kill the cancer stem cells and one has to wait until the transit-amplifying cells become senescent. In these cases, a combination therapy of anti-cancer stem cell drugs and classical chemotherapy would be favorable.

Research in the field of anti-cancer stem cell drugs is in progress, but only time will tell if scientists will be able to successfully develop these highly specific drugs. Currently, the impact of cyclopamin, a natural product from the plant veratrum californicum, on glioblastoma cancer stem cells is studied (77). The hitherto existing problems in glioblastoma therapy are probably also due to the fact that current chemoand radiotherapies do not affect cancer stem cells. Cyclopamin in contrast inhibits the 'hedgehog'-signaling pathway and hence kills the cancer stem cells. Considering recent progress in that field, it might be hoped that further research will establish a basis for the development of several highly specific drugs against cancer stem cells.

\section{Conclusion}

The molecular principles of cancer invasion and metastasis are at the same time highly complex and essential for a profound understanding of carcinogenesis. Moreover, the topic is immensely relevant since $90 \%$ of all cancer deaths can be attributed to metastases and not to the primary tumors. The currently applied therapy approaches mainly focus on rapidly proliferating cells. New insights into the molecular processes of invasion and metastasis as well as the concept of cancer stem cells may pave the way for new, highly specific and successful drugs. The precondition for this purpose is further research in the field for a better understanding of these processes.

\section{References}

1. Hahn WC and Weinberg RA: Rules for making human tumor cells. N Engl J Med 347: 1593-1603, 2002.

2. Lynch TJ, Bell DW, Sordella R, et al: Activating mutations in the epidermal growth factor receptor underlying responsiveness of non-small-cell lung cancer to gefitinib. N Engl J Med 350: 2129-2139, 2004.

3. Rajalingam K, Schreck R, Rapp UR and Albert S: Ras oncogenes and their downstream targets. Biochim Biophys Acta 1773: 1177-1195, 2007.

4. Wieser RJ, Renauer D, Schafer A, et al: Growth control in mammalian cells by cell-cell contacts. Environ Health Perspect 88: 251-253, 1990 .

5. Lane DP: Cancer. p53, guardian of the genome. Nature 358: $15-16,1992$.

6. Hollstein M, Sidransky D, Vogelstein B and Harris CC: p53 mutations in human cancers. Science 253: 49-53, 1991

7. Tovar C, Rosinski J, Filipovic Z, et al: Small-molecule MDM2 antagonists reveal aberrant p53 signaling in cancer: implications for therapy. Proc Natl Acad Sci USA 103: 1888-1893, 2006.

8. Malkin D, Li FP, Strong LC, et al: Germ line p53 mutations in a familial syndrome of breast cancer, sarcomas, and other neoplasms. Science 250: 1233-1238, 1990

9. Mukae N, Enari M, Sakahira H, et al: Molecular cloning and characterization of human caspase-activated DNase. Proc Natl Acad Sci USA 95: 9123-9128, 1998.

10. Segal M, Niazi S, Simons MP, Galati SA and Zangrilli JG: Bid activation during induction of extrinsic and intrinsic apoptosis in eosinophils. Immunol Cell Biol 85: 518-524, 2007.

11. Igney FH and Krammer PH: Tumor counterattack: fact or fiction? Cancer Immunol Immunother 54: 1127-1136, 2005.

12. Auguste P, Lemiere S, Larrieu-Lahargue F and Bikfalvi A: Molecular mechanisms of tumor vascularization. Crit Rev Oncol Hematol 54: 53-61, 2005.

13. Brantley DM, Cheng N, Thompson EJ, et al: Soluble Eph A receptors inhibit tumor angiogenesis and progression in vivo. Oncogene 21: 7011-7026, 2002.

14. Hayflick L: The limited in vitro lifetime of human diploid cell strains. Exp Cell Res 37: 614-636, 1965.

15. Zhang X, Mar V, Zhou W, Harrington L and Robinson MO: Telomere shortening and apoptosis in telomerase-inhibited human tumor cells. Genes Dev 13: 2388-2399, 1999.

16. Bodnar AG, Ouellette M, Frolkis M, et al: Extension of lifespan by introduction of telomerase into normal human cells. Science 279: 349-352, 1998

17. Dimri GP, Lee X, Basile G, et al: A biomarker that identifies senescent human cells in culture and in aging skin in vivo. Proc Natl Acad Sci USA 92: 9363-9367, 1995.

18. Cohen SB, Graham ME, Lovrecz GO, Bache N, Robinson PJ and Reddel RR: Protein composition of catalytically active human telomerase from immortal cells. Science 315: 1850-1853, 2007.

19. Herbert B, Pitts AE, Baker SI, et al: Inhibition of human telomerase in immortal human cells leads to progressive telomere shortening and cell death. Proc Natl Acad Sci USA 96: 14276-14281, 1999.

20. Hahn WC, Stewart SA, Brooks MW, et al: Inhibition of telomerase limits the growth of human cancer cells. Nat Med 5: 1164-1170, 1999 . 
21. Wang ES, Wu K, Chin AC, et al: Telomerase inhibition with an oligonucleotide telomerase template antagonist: in vitro and in vivo studies in multiple myeloma and lymphoma. Blood 103: 258-266, 2004.

22. Burger AM, Dai F, Schultes CM, et al: The G-quadruplexinteractive molecule BRACO-19 inhibits tumor growth, consistent with telomere targeting and interference with telomerase function. Cancer Res 65: 1489-1496, 2005.

23. Muntoni A and Reddel RR: The first molecular details of ALT in human tumor cells. Hum Mol Genet 14: R191-R196, 2005.

24. Mehlen P and Puisieux A: Metastasis: a question of life or death. Nat Rev Cancer 6: 449-458, 2006.

25. Luzzi KJ, MacDonald IC, Schmidt EE, et al: Multistep nature of metastatic inefficiency: dormancy of solitary cells after successful extravasation and limited survival of early micrometastases. Am J Pathol 153: 865-873, 1998.

26. Weinberg RA: The Biology of Cancer: Garland Science 2006.

27. Christofori G: Changing neighbours, changing behaviour: cell adhesion molecule-mediated signalling during tumour progression. EMBO J 22: 2318-2323, 2003.

28. Herzig M, Savarese F, Novatchkova M, Semb H and Christofori G: Tumor progression induced by the loss of E-cadherin independent of beta-catenin/Tcf-mediated Wnt signaling. Oncogene 26: 2290-2298, 2007.

29. Beavon IR: The E-cadherin-catenin complex in tumour metastasis: structure, function and regulation. Eur J Cancer 36: 1607-1620, 2000.

30. Bolos V, Peinado H, Perez-Moreno MA, Fraga MF, Esteller M and Cano A: The transcription factor Slug represses E-cadherin expression and induces epithelial to mesenchymal transitions: a comparison with Snail and E47 repressors. J Cell Sci 116: 499-511, 2003

31. Richards FM, McKee SA, Rajpar MH, et al: Germline E-cadherin gene $(\mathrm{CDH} 1)$ mutations predispose to familial gastric cancer and colorectal cancer. Hum Mol Genet 8: 607-610, 1999.

32. http://www.fennerlabor.de/Cytokeratine_als_Tum.281.0.html (accessed date: March 10th 2008).

33. Gordon MD and Nusse R: Wnt signaling: multiple pathways, multiple receptors, and multiple transcription factors. J Biol Chem 281: 22429-22433, 2006

34. Miller JR, Hocking AM, Brown JD and Moon RT: Mechanism and function of signal transduction by the Wnt/beta-catenin and Wnt/Ca2 ${ }^{+}$pathways. Oncogene 18: 7860-7872, 1999.

35. Rosivatz E, Becker I, Specht K, et al: Differential expression of the epithelial-mesenchymal transition regulators snail, SIP1, and twist in gastric cancer. Am J Pathol 161: 1881-1891, 2002.

36. Yang J, Mani SA, Donaher JL, et al: Twist, a master regulator of morphogenesis, plays an essential role in tumor metastasis. Cell 117: 927-939, 2004.

37. Yang J, Mani SA and Weinberg RA: Exploring a new twist on tumor metastasis. Cancer Res 66: 4549-4552, 2006.

38. Huber MA, Azoitei N, Baumann B, et al: NF-kappaB is essential for epithelial-mesenchymal transition and metastasis in a model of breast cancer progression. J Clin Invest 114: 569-581, 2004

39. Wyckoff J, Wang W, Lin EY, et al: A paracrine loop between tumor cells and macrophages is required for tumor cell migration in mammary tumors. Cancer Res 64: 7022-7029, 2004.

40. Wang W, Goswami S, Sahai E, Wyckoff JB, Segall JE and Condeelis JS: Tumor cells caught in the act of invading: their strategy for enhanced cell motility. Trends Cell Biol 15: 138-145, 2005.

41. Lin EY, Nguyen AV, Russell RG and Pollard JW: Colonystimulating factor 1 promotes progression of mammary tumors to malignancy. J Exp Med 193: 727-740, 2001.

42. Lu Z, Jiang G, Blume-Jensen P and Hunter T: Epidermal growth factor-induced tumor cell invasion and metastasis initiated by dephosphorylation and downregulation of focal adhesion kinase. Mol Cell Biol 21: 4016-4031, 2001.

43. Rudzki Z and Jothy S: CD44 and the adhesion of neoplastic cells. Mol Pathol 50: 57-71, 1997.

44. Isacke CM and Yarwood H: The hyaluronan receptor, CD44. Int J Biochem Cell Biol 34: 718-721, 2002.

45. Stauder R, Eisterer W, Thaler J and Gunthert U: CD44 variant isoforms in non-Hodgkin's lymphoma: a new independent prognostic factor. Blood 85: 2885-2899, 1995

46. Efferth T: Molekulare Pharmakologie und Toxikologie: Springer Publishing, Berlin, Heidelberg, 2006.

47. Overall CM and Lopez-Otin C: Strategies for MMP inhibition in cancer: innovations for the post-trial era. Nat Rev Cancer 2: $657-672,2002$
48. Blasi F and Carmeliet P: uPAR: a versatile signalling orchestrator. Nat Rev Mol Cell Biol 3: 932-943, 2002.

49. Hall A: Rho GTPases and the actin cytoskeleton. Science 279: 509-514, 1998.

50. Frederick MJ and Clayman GL: Chemokines in cancer. Expert Rev Mol Med 3: 1-18, 2001

51. Wong SY and Hynes RO: Lymphatic or hematogenous dissemination: how does a metastatic tumor cell decide? Cell Cycle 5: 812-817, 2006

52. Muller A, Homey B, Soto H, et al: Involvement of chemokine receptors in breast cancer metastasis. Nature 410: 50-56, 2001.

53. Arenberg DA, Kunkel SL, Polverini PJ, Glass M, Burdick MD and Strieter RM: Inhibition of interleukin-8 reduces tumorigenesis of human non-small cell lung cancer in SCID mice. J Clin Invest 97: 2792-2802, 1996.

54. Giovarelli M, Cappello P, Forni G, et al: Tumor rejection and immune memory elicited by locally released LEC chemokine are associated with an impressive recruitment of APCs, lymphocytes, and granulocytes. J Immunol 164: 3200-3206, 2000.

55. Fujisawa N, Sakao Y, Hayashi S, Hadden WA III, Harmon CL and Miller EJ: alpha-chemokine growth factors for adenocarcinomas; a synthetic peptide inhibitor for alpha-chemokines inhibits the growth of adenocarcinoma cell lines. J Cancer Res Clin Oncol 126: 19-26, 2000.

56. Smets FN, Chen Y, Wang LJ and Soriano HE: Loss of cell anchorage triggers apoptosis (anoikis) in primary mouse hepatocytes. Mol Genet Metab 75: 344-352, 2002

57. Mueller BM, Reisfeld RA, Edgington TS and Ruf W: Expression of tissue factor by melanoma cells promotes efficient hematogenous metastasis. Proc Natl Acad Sci USA 89: 11832-11836, 1992.

58. Schluter K, Gassmann P, Enns A, et al: Organ-specific metastatic tumor cell adhesion and extravasation of colon carcinoma cells with different metastatic potential. Am J Pathol 169: 1064-1073, 2006.

59. Janeway CA, Walport M and Shlomchik M: Immunobiology. 6th edition. Taylor and Francis Group, Abingdon, Oxford, 2005.

60. Chen C, Pore N, Behrooz A, Ismail-Beigi F and Maity A: Regulation of glut 1 mRNA by hypoxia-inducible factor-1. Interaction between H-ras and hypoxia. J Biol Chem 276: 95199525, 2001

61. Kind KL, Collett RA, Harvey AJ and Thompson JG: Oxygenregulated expression of GLUT-1, GLUT-3, and VEGF in the mouse blastocyst. Mol Reprod Dev 70: 37-44, 2005.

62. Sauter BV, Martinet O, Zhang WJ, Mandeli J and Woo SL: Adenovirus-mediated gene transfer of endostatin in vivo results in high level of transgene expression and inhibition of tumor growth and metastases. Proc Natl Acad Sci USA 97: 4802-4807, 2000.

63. Steeg PS, Cohn KH and Leone A: Tumor metastasis and nm23: current concepts. Cancer Cells 3: 257-262, 1991.

64. Streuli CH: Maspin is a tumour suppressor that inhibits breast cancer tumour metastasis in vivo. Breast Cancer Res 4: 137-140, 2002.

65. Rinker-Schaeffer CW, O'Keefe JP, Welch DR and Theodorescu D: Metastasis suppressor proteins: discovery, molecular mechanisms, and clinical application. Clin Cancer Res 12: 3882-3889, 2006.

66. Fialkow PJ: The origin and development of human tumors studied with cell markers. N Engl J Med 291: 26-35, 1974.

67. Novelli M, Cossu A, Oukrif D, et al: X-inactivation patch size in human female tissue confounds the assessment of tumor clonality. Proc Natl Acad Sci USA 100: 3311-3314, 2003.

68. Mapara MY, Kim YM, Marx J and Sykes M: Donor lymphocyte infusion-mediated graft-versus-leukemia effects in mixed chimeras established with a non-myeloablative conditioning regimen: extinction of graft-versus-leukemia effects after conversion to full donor chimerism. Transplantation 76: 297-305, 2003.

69. Sagar J, Chaib B, Sales K, Winslet M and Seifalian A: Role of stem cells in cancer therapy and cancer stem cells: a review. Cancer Cell Int 7: 9, 2007.

70. Bernstein A: Cancer stem cells: the centrality of translational research to cancer control. CMAJ 176: 29-30, 2007.

71. Reya T, Morrison SJ, Clarke MF and Weissman IL: Stem cells, cancer, and cancer stem cells. Nature 414: 105-111, 2001.

72. Waterworth A: Introducing the concept of breast cancer stem cells. Breast Cancer Res 6: 53-54, 2004. 
73. Prince ME, Sivanandan R, Kaczorowski A, et al: Identification of a subpopulation of cells with cancer stem cell properties in head and neck squamous cell carcinoma. Proc Natl Acad Sci USA 104: 973-978, 2007.

74. Zhang P, Gao WY, Turner S and Ducatman BS: Gleevec (STI-571) inhibits lung cancer cell growth (A549) and potentiates the cisplatin effect in vitro. Mol Cancer 2: 1, 2003.

75. Lagasse E: Cancer stem cells with genetic instability: the best vehicle with the best engine for cancer. Gene Ther 15: 136-142, 2008.
76. Lou H and Dean M: Targeted therapy for cancer stem cells: the patched pathway and ABC transporters. Oncogene 26: 1357-1360, 2007.

77. Bar EE, Chaudhry A, Lin A, et al: Cyclopamine-mediated hedgehog pathway inhibition depletes stem-like cancer cells in glioblastoma. Stem Cells 25: 2524-2533, 2007.

78. Efferth T, Löhrke H and Volm M: Reciprocal correlation between expression of P-glycoprotein and accumulation of rhodamine 123 in human tumors. Anticancer Res 9: 1633-1637, 1989. 